\title{
Five Texture Zeros for Dirac Neutrino Mass Matrices
}

\author{
Richard H. Benavides, ${ }^{1, *}$ Yithsbey Giraldo, ${ }^{2, \dagger}$ Luis Muñoz, ${ }^{1, \ddagger}$ William A. Ponce,,${ }^{3, \S}$ and Eduardo Rojas ${ }^{2, \uparrow}$ \\ 1 Facultad de Ciencias Exactas y Aplicadas, Instituto Tecnológico Metropolitano, \\ Calle 73 No 76 A - 354, Vía el Volador, Medellín, Colombia \\ ${ }^{2}$ Departamento de Física, Universidad de Nariño, A.A. 1175, San Juan de Pasto, Colombia \\ ${ }^{3}$ Instituto de Física, Universidad de Antioquia, Calle 70 No. 52-21, Apartado Aéreo 1226, Medellín, Colombia
}

\begin{abstract}
In this work we propose new five textures zeros for the mass matrices in the lepton sector in order to predict values for the neutrino masses. In our approach we go beyond the Standard Model by assuming Dirac masses for the neutrinos, feature which allows us to make a theoretical prediction for the lightest neutrino mass in the normal ordering. The textures analyzed have enough free parameters to adjust the $V_{\mathrm{PMNS}}$ mixing matrix including the $\mathrm{CP}$-violating phase, the neutrino mass squared differences $\delta m_{21}^{2}, \delta m_{31}^{2}$, and the three charged lepton masses. In order to have reliable results two different approaches are used: the first one is based on a least-squares analysis to fit the lepton masses and the mixing parameters to their corresponding experimental values, for this case the best fit for the lightest neutrino mass is $\left(3.9 \pm_{0.8}^{0.6}\right) \times 10^{-3} \mathrm{eV}$; the second approach is just algebraic, based on the weak basis transformation method, in this case the lightest neutrino mass consistent with the experimental values and the restrictions coming from the five texture zeros of the mass matrices is equal to $(3.5 \pm 0.9) \times 10^{-3} \mathrm{eV}$.
\end{abstract}

PACS numbers: 14.60.Pq, 14.60.St, 14.60.Lm.

\section{INTRODUCTION}

The Standard Model (SM) of the strong and electroweak interactions [1] based on the local gauge group $S U(3)_{c} \otimes S U(2)_{L} \otimes U(1)_{Y}$, with the unbroken $S U(3)_{c}$ sector confining and the electroweak sector $S U(2)_{L} \otimes U(1)_{Y}$ spontaneously broken by one scalar complex Higgs doublet down to $U(1)_{e m}$, has been very successful in explaining several experimental issues. Unanswered questions of the model are the explanation of the total number of families present in nature, the hierarchy of the charged fermion mass spectrum, the quark and lepton mixing angles and the origin of $\mathrm{CP}$ violation. Even mayor issues are the existence of dark energy and dark matter and the smallest of the neutrino masses and their oscillations.

The extension of the SM with three right handed neutrinos provides with several nice features: a) it allows the introduction of nine additional complex Dirac mass terms in the neutral lepton sector [2], b) it permits the implementation of the seesaw mechanism [3-8], and c) it conduces to an analysis with hermitian mass matrices in the lepton sector of the model. Although the first two features have been widely explored in the literature, the last one has not; it is the purpose of this paper to analyze the mathematical and numerical consequences of this last fact.

The texture of the Dirac neutrinos mass matrix has been the subject of several recent studies [9-19]; this, in part, has been motivated by the non-observation of the double beta decay [20]. As it is well known, the existing experiments have not been able to determine if neutrinos are Majorana or Dirac particles; however, most work on these topics assumes that neutrinos are Majorana, whereas the case for Dirac neutrino masses has not been studied properly. In this analysis we assume that neutrinos are Dirac particles [2], which allows us to use the weak basis transformation, the polar decomposition theorem, and the most recent experimental data to make predictions based on a given texture for the lepton mass matrices. It is interesting to determine the consequences of a given texture on the prediction of the neutrino masses; indeed, for Majorana masses there are several works predicting a mass of a few milli-electron volts for the lightest neutrino mass [21-23]; which, as we will see, has the same order of magnitude as the results presented in this work.

There exist top-bottom motivations to assume Dirac masses for the neutrinos; in models derived from string theory the Majorana masses are suppressed by selection rules related to the underlying symmetries [24, 25]. In these models, Majorana masses can also be generated by active neutrinos via gravitational effects; however, these masses are very

\footnotetext{
*Electronic address: richardbenavides@itm.edu.co

${ }^{\dagger}$ Electronic address: yithsbey@gmail.com

${ }^{\ddagger}$ Electronic address: luismunoz@itm.edu.co

$\S$ Electronic address: william.ponce@udea.edu.co

『Electronic address: eduro4000@gmail.com
} 
small, even compared with the small scales in neutrino physics [26, 27]. The Dirac neutrino electromagnetic properties are quite testable too due to the magnetic dipole moment which is different from zero at the quantum level [28]. The same is not true for Majorana neutrinos, where their electromagnetic properties are reduced because they are their own antiparticles. In this way, theoretical models with Dirac neutrinos are a motivation to refine the electromagnetic constraints on the neutrino properties.

In the SM extended with three right-handed neutrinos (SMRHN) and forbidding the bare Majorana masses of the right-handed neutral states (obtained just by assuming lepton number conservation), the lepton mass terms after the spontaneous breaking of the local symmetry are given by

$$
-\mathcal{L}_{D}=\bar{\nu}_{L} M_{n} \nu_{R}+\bar{\ell}_{L} M_{\ell} \ell_{R}+\text { h.c, }
$$

where $\nu_{L, R}=\left(\nu_{e}, \nu_{\mu}, \nu_{\tau}\right)_{L, R}^{T}$ and $\ell_{L, R}=(e, \mu, \tau)_{L, R}^{T}$ (the upper $T$ stands for transpose). The matrices $M_{n}$ and $M_{\ell}$ in (1) are in general $3 \times 3$ complex mass matrices. In the most general case they contain 36 free parameters. In the context of the SM extended with three right-handed neutrinos, such a large number of parameters can be drastically cut by making use of the polar theorem of matrix algebra, by which, one can always decompose a general complex matrix as the product of an hermitian times a unitary matrix. Since in the context of the SM extended with three right-handed neutrinos the unitary matrix can be absorbed in a redefinition of the right handed lepton components, this immediately brings the number of free parameters from 36 down to 18 (the other eighteen parameters can be hidden in the right-handed lepton components in the context of these models and some of its extensions, but not in its left-right symmetric extensions). So, as far as this model is concerned, we may treat without loss of generality $M_{n}$ and $M_{\ell}$ as two hermitian mass matrices, with 18 real parameters in total, out of which six are phases. Since five of those phases can be absorbed in a redefinition of the lepton fields $[29,30]$, the total number of free parameters we may play with in $M_{n}$ and $M_{\ell}$ are 12 real parameters and one phase; this last one used to explain the CP violation phenomena.

Now, in the context of the SMRHN, it is always possible to implement the so called weak basis (WB) transformation [31,32], which leaves the two $3 \times 3$ lepton mass matrices Hermitian and does not alter the physics implicit in the weak currents. Such a WB transformation is a unitary transformation acting simultaneously in the neutral and lepton fermion mass matrices [31,32]. That is

$$
M_{n} \rightarrow M_{n}^{R}=U M_{n} U^{\dagger}, \quad M_{l} \rightarrow M_{l}^{R}=U M_{n} U^{\dagger},
$$

where $U$ is an arbitrary unitary matrix. We say then that the two representations $\left(M_{n}, M_{l}\right)$ and $\left(M_{n}^{R}, M_{l}^{R}\right)$ are equivalent in the sense that they are related to the same $V_{\text {PMNS }}$ mixing matrix. This kind of transformation plays an important role in the study of the so-called flavor problem.

In references $[31,32]$ it was shown that, for hermitian quark mass matrices it is always possible to perform a weak basis transformation, in such a way that it is always possible to end up with two hermitian mass matrices with three texture zeros which do not have any physical implication. As we mentioned above we extend the SM to include the right-handed neutrinos and consider them to be Dirac type particles. Thus, the mechanism implemented in the quark sector can be transferred, with few modifications, directly to the lepton sector. With these three non-physical texture zeros the number of free parameters in $M_{n}^{R}$ and $M_{\ell}^{R}$ reduces from twelve to nine real parameters and one phase, just enough to fit the physical values for the six Dirac lepton masses, the three mixing angles, and the CP violation phase. Any extra texture zero can only be a physical assumption and should imply a relationship between the ten physical parameters. But for the case of neutrinos, their masses are not known, and just two square mass differences are experimentally available; that is: $\delta m_{21}=m_{2}^{2}-m_{1}^{2}, \delta m_{31}=m_{3}^{2}-m_{1}^{2}$. Therefore, only two experimental restrictions are present in the analysis of the neutral lepton sector (which is not the case in the quark sector), and a fourth texture zero, if possible, will not produce a testable prediction at all. The next step, a fifth texture zero should provide us with a physical prediction and a possible sixth texture zero will provide with two. (Counting degrees of freedom we find that the maximum number of such texture zeros consistent with the absence of a zero mass eigenvalue and a nondegenerate mass spectrum in the lepton sector is just six, with three in the neutral sector and three in the charged one.)

In the analysis which follows we are going to use the SM ingredients including one single complex Higgs doublet, enlarged with three right-handed neutrinos (one for each family) in order to provide the neutral sector with Dirac masses, and an unknown symmetry able to produce five texture zeros in the lepton sector. Two cases are going to be considered: one with three texture zeros in the neutral lepton sector and two in the charged one, and a second case with two texture zeros in the neutral lepton sector and three in the charged one. Two different analysis are implemented, one for each situation. Analytic and numeric results are presented, taking special care to accommodate the latest experimental data available [33], including the CP violation phenomena. 


\section{FIVE TEXTURE ZEROS. FIRST CASE.}

In the context of the SMRHN with lepton number conservation, in the weak basis, and after breaking the local gauge symmetry, the Lagrangian mass term for the lepton sector is given by

$$
-\mathcal{L}_{D}=\bar{\nu}_{L}^{\prime} M_{n}^{\prime} \nu_{R}^{\prime}+\bar{\nu}_{R}^{\prime} M_{n}^{\prime \dagger} \nu_{L}^{\prime}+\bar{\ell}_{L}^{\prime} M_{\ell}^{\prime} \ell_{R}^{\prime}+\bar{\ell}_{R}^{\prime} M_{\ell}^{\prime \dagger} \ell_{L}^{\prime}
$$

where $M_{n}^{\prime}$ and $M_{\ell}^{\prime}$ are the neutrino and charged lepton mass matrices respectively (primed fields and matrices refer to the weak basis).

Let us now assume that for a given symmetry, the Hermitian mass matrices $M_{n}^{\prime}$ and $M_{\ell}^{\prime}$ present the following textures

$$
M_{n}^{\prime}=\left(\begin{array}{ccc}
c_{n} & a_{n} & 0 \\
a_{n}^{*} & 0 & b_{n} \\
0 & b_{n}^{*} & 0
\end{array}\right), \quad M_{\ell}^{\prime}=\left(\begin{array}{ccc}
0 & a_{\ell} & 0 \\
a_{\ell}^{*} & d_{\ell} & b_{\ell} \\
0 & b_{\ell}^{*} & c_{\ell}
\end{array}\right)
$$

In what follows we are going to analyze the consequences of this particular pattern with three texture zeros in the neutral sector and two in the charged one.

The first step is to remove the phases; this can be done by the following unitary transformation:

$$
M_{n, \ell}^{\prime}=\lambda_{n, \ell}^{\dagger} M_{n, \ell} \lambda_{n, \ell}
$$

which is achieved by using the diagonal matrices $\lambda_{n}=\left(1, e^{i \alpha_{n_{1}}}, e^{i \alpha_{n_{1}}+i \alpha_{n_{2}}}\right)$ and $\lambda_{\ell}=\left(1, e^{i \alpha_{\ell_{1}}}, e^{i \alpha_{\ell_{1}}+i \alpha_{\ell_{2}}}\right)$, respectively, and $M_{n, \ell}$ are the matrices whose components are the absolute values of the corresponding entries in $M_{n, \ell}^{\prime}$ (i.e., $\left.\left(M_{n, \ell}\right)_{i, j}=\left|\left(M_{n, \ell}^{\prime}\right)_{i, j}\right|\right)$. If we rotate these matrices by using the orthogonal transformation $R_{n, \ell}\left(R_{n, \ell}^{T} R_{n, \ell}=\mathbb{1}\right)$ to the mass eigenstate space (the physical basis), we get

$$
M_{n, \ell}^{\prime}=\lambda_{n, \ell}^{\dagger} R_{n, \ell}^{T}\left(\begin{array}{ccc}
m_{1, e} & 0 & 0 \\
0 & -m_{2, \mu} & 0 \\
0 & 0 & m_{3, \tau}
\end{array}\right) R_{n, \ell} \lambda_{n, \ell} \equiv U_{n, \ell} M_{n, \ell}^{\operatorname{diag}} U_{n, \ell}^{\dagger}
$$

where at least a negative eigenvalue is needed in order to generate a texture-zero in the diagonal [32, 34-36]. Here $m_{1}, m_{2}$ and $m_{3}$ are the masses of the electron, muon and tau neutrinos respectively, with the masses of the charged leptons given by: $m_{e}=0.5109989461 \pm 0.0000000031, m_{\mu}=105.6583745 \pm 0.0000024$ and $m_{\tau}=1776.86 \pm 0.12$, which correspond to the electron, muon and tau masses, respectively (in MeV) [33]. After rotating to the mass eigenstates the eigenvalues can be positive, negative or zero. In these expressions $M_{n}^{\text {diag }}$ and $M_{\ell}^{\text {diag }}$ are the diagonal mass matrices for the neutrino and charged lepton sectors respectively. In accord with the standard notation we use $U_{n} \equiv\left(R_{n} \lambda_{n}\right)^{\dagger}$ and $U_{\ell} \equiv\left(R_{\ell} \lambda_{\ell}\right)^{\dagger}$, two unitary matrices used to rotate from the weak basis to the physical basis. From equations (3) and (4) we obtain the relation between the states in the mass basis $\nu_{L, R}, \ell_{L, R}$ and the corresponding states in the interaction basis $\nu_{L, R}^{\prime}, \ell_{L, R}^{\prime}$ :

$$
\nu_{L, R}^{\prime}=U_{n} \nu_{L, R}, \quad \ell_{L, R}^{\prime}=U_{\ell} \ell_{L, R}
$$

Replacing these expressions in the lepton sector of the weak current we obtain

$$
\mathcal{L}_{W^{-}}=-\frac{g}{\sqrt{2}} W^{-} \bar{\ell}_{L}^{\prime} \gamma^{\mu} \nu_{L}^{\prime}+\text { h.c }=-\frac{g}{\sqrt{2}} W^{-} \bar{\ell}_{L} \gamma^{\mu} U_{\ell}^{\dagger} U_{n} \nu_{L}+\text { h.c }
$$

in such a way that the Pontecorvo-Maki-Nakagawa-Sakata matrix (PMNS matrix) is given by

$$
V_{\mathrm{PMNS}}=U_{\ell}^{\dagger} U_{n}=R_{\ell} \Phi R_{n}^{T}
$$

where $\Phi=\lambda_{\ell} \lambda_{n}^{\dagger}$ is a diagonal phase matrix. For the neutrino mass matrix normal ordering is assumed [37], i.e.: $m_{3}>m_{2}>m_{1}$, where: $m_{2}^{2}=m_{1}^{2}+\delta m_{21}^{2}$, and $m_{3}^{2}=m_{1}^{2}+\delta m_{31}^{2}$, with $\delta m_{21}^{2}, \delta m_{31}^{2}>0$ [38].

By imposing the invariance of the trace and the determinant of the mass $\operatorname{matrices}\left(\operatorname{tr}\left[M_{n, \ell}^{\prime}\right]=\operatorname{tr}\left[M_{n, \ell}^{\operatorname{diag}}\right]\right.$, $\operatorname{tr}\left[\left(M_{n, \ell}^{\prime}\right)^{2}\right]=\operatorname{tr}\left[\left(M_{n, \ell}^{\text {diag }}\right)^{2}\right]$, and $\left.\operatorname{Det}\left[M_{n, \ell}^{\prime}\right]=\operatorname{Det}\left[M_{n, \ell}^{\text {diag }}\right]\right)$, the following relations are obtained for this particular texture: 


$$
\begin{aligned}
c_{n} & =m_{1}-m_{2}+m_{3}, & d_{\ell} & =m_{e}-m_{\mu}+m_{\tau}-c_{\ell}, \\
\left|a_{n}\right| & =\sqrt{\frac{\left(m_{1}-m_{2}\right)\left(m_{1}+m_{3}\right)\left(m_{2}-m_{3}\right)}{m_{1}-m_{2}+m_{3}}}, & \left|b_{\ell}\right| & =\sqrt{\frac{\left(c_{\ell}-m_{\ell}\right)\left(c_{\ell}+m_{\mu}\right)\left(m_{\tau}-c_{\ell}\right)}{c_{\ell}},} \\
\left|b_{n}\right| & =\sqrt{\frac{m_{1} m_{2} m_{3}}{m_{1}-m_{2}+m_{3}}}, & \left|a_{\ell}\right| & =\sqrt{\frac{m_{e} m_{\mu} m_{\tau}}{c_{\ell}}} .
\end{aligned}
$$

From the previous identifications, it is possible to obtain an explicit form for the mass matrices of leptons that allows us to obtain, through diagonalization of $M_{n}$ and $M_{\ell}$, the orthogonal matrices in Eq. (9),

$$
\begin{aligned}
& R_{n}=\left(\begin{array}{cccc}
-\sqrt{\frac{m_{1}\left(m_{2}-m_{1}\right)\left(m_{1}+m_{3}\right)}{\left(m_{1}+m_{2}\right)\left(m_{3}-m_{1}\right)\left(m_{1}-m_{2}+m_{3}\right)}} & \sqrt{\frac{m_{1}\left(m_{3}-m_{2}\right)}{\left(m_{1}+m_{2}\right)\left(m_{3}-m_{1}\right)}} & \sqrt{\frac{m_{2} m_{3}\left(m_{3}-m_{2}\right)}{\left(m_{1}+m_{2}\right)\left(m_{3}-m_{1}\right)\left(m_{1}-m_{2}+m_{3}\right)}} \\
\sqrt{\frac{m_{2}\left(m_{1}-m_{2}\right)\left(m_{2}-m_{3}\right)}{\left(m_{1}+m_{2}\right)\left(m_{2}+m_{3}\right)\left(m_{1}-m_{2}+m_{3}\right)}} & -\sqrt{\frac{m_{2}\left(m_{1}+m_{3}\right)}{\left(m_{1}+m_{2}\right)\left(m_{2}+m_{3}\right)}} & \sqrt{\frac{m_{1} m_{3}\left(m_{1}+m_{3}\right)}{\left(m_{1}+m_{2}\right)\left(m_{2}+m_{3}\right)\left(m_{1}-m_{2}+m_{3}\right)}} \\
\sqrt{\frac{m_{3}\left(m_{1}+m_{3}\right)\left(m_{3}-m_{2}\right)}{\left(m_{3}-m_{1}\right)\left(m_{2}+m_{3}\right)\left(m_{1}-m_{2}+m_{3}\right)}} & \sqrt{\frac{m_{3}\left(m_{2}-m_{1}\right)}{\left(m_{2}+m_{3}\right)\left(m_{3}-m_{1}\right)}} & \sqrt{\frac{m_{1} m_{2}\left(m_{2}-m_{1}\right)}{\left(m_{3}-m_{1}\right)\left(m_{2}+m_{3}\right)\left(m_{1}-m_{2}+m_{3}\right)}}
\end{array}\right), \\
& R_{\ell}=\left(\begin{array}{cccc}
-\sqrt{\frac{m_{\mu} m_{\tau}\left(c_{\ell}-m_{e}\right)}{c_{\ell}\left(m_{e}+m_{\mu}\right)\left(m_{\tau}-m_{e}\right)}} & -\sqrt{\frac{m_{e}\left(c_{\ell}-m_{e}\right)}{\left(m_{e}+m_{\mu}\right)\left(m_{\tau}-m_{e}\right)}} & \sqrt{\frac{m_{e}\left(c_{\ell}+m_{\mu}\right)\left(c_{\ell}-m_{\tau}\right)}{c_{\ell}\left(m_{e}+m_{\mu}\right)\left(m_{e}-m_{\tau}\right)}} \\
\sqrt{\frac{m_{e} m_{\tau}\left(c_{\ell}+m_{\mu}\right)}{c_{\ell}\left(m_{e}+m_{\mu}\right)\left(m_{\mu}+m_{\tau}\right)}} & -\sqrt{\frac{m_{\mu}\left(c_{\ell}+m_{\mu}\right)}{\left(m_{e}+m_{\mu}\right)\left(m_{\mu}+m_{\tau}\right)}} & \sqrt{\frac{m_{\mu}\left(m_{e}-c_{\ell}\right)\left(c_{\ell}-m_{\tau}\right)}{c_{\ell}\left(m_{e}+m_{\mu}\right)\left(m_{\mu}+m_{\tau}\right)}} \\
\sqrt{\frac{m_{e} m_{\mu}\left(c_{\ell}-m_{\tau}\right)}{c_{\ell}\left(m_{e}-m_{\tau}\right)\left(m_{\mu}+m_{\tau}\right)}} & \sqrt{\frac{m_{\tau}\left(m_{\tau}-c_{\ell}\right)}{\left(m_{\tau}-m_{e}\right)\left(m_{\mu}+m_{\tau}\right)}} & \sqrt{\frac{m_{\tau}\left(c_{\ell}-m_{e}\right)\left(c_{\ell}+m_{\mu}\right)}{c_{\ell}\left(m_{\tau}-m_{e}\right)\left(m_{\mu}+m_{\tau}\right)}}
\end{array}\right) .
\end{aligned}
$$

The entries of $R_{n}$ and in $R_{\ell}$ are real values because of the normal hierarchy assumed in neutrino masses, and to the hierarchy of the charged lepton sector $m_{\tau}>m_{\mu}>m_{e}$, with this in mind the constraint for $c_{\ell}$ is established to be in the interval $m_{e}<c_{\ell}<m_{\tau}$. A closer look to the former expressions shows that for this analysis $m_{1}$ and $c_{\ell}$ can be taken as free parameters to be fixed by an statistical analysis.

\section{A. Least squares analysis}

From equation (9) we have that $V_{\mathrm{PMNS}}=R_{\ell} \Phi R_{n}^{T}$, with $\Phi$ the following diagonal matrix:

$$
\Phi=\left(\begin{array}{ccc}
1 & 0 & 0 \\
0 & e^{i \phi_{1}} & 0 \\
0 & 0 & e^{i \phi_{2}}
\end{array}\right),
$$

where $\phi_{1}$ and $\phi_{2}$ are free parameters.

Then, the former analysis imply that the matrix $V_{\mathrm{PMNS}}$ is a function of the free parameters $\left(m_{1}, c_{\ell}, \phi_{1}, \phi_{2}\right)$ where we have chosen $m_{1}$ as the lightest neutrino mass. After a numerical adjustment by means of the $\chi^{2}$ analysis, it is found that for our particular choice of the five-zero texture lepton mass matrices in Eq. (4), the normal hierarchy is favored. Neglecting correlations, the $\chi^{2}$ function is given by

$$
\chi^{2}=P_{J}^{2}+\sum_{i, j=1,2,3} P_{i j}^{2},
$$

where the pulls are

$$
P_{i j}=\frac{U_{i j}-\bar{U}_{i j}}{\delta U_{i j}}
$$

where $U_{i j}=\left|\left(V_{\mathrm{PMNS}}\right)_{i j}\right|$ is the absolute value of the components from the product of the diagonalization matrices (9), the absolute values $\bar{U}_{i j}$ correspond to the global averages for the components of the PMNS matrix and $\delta U_{i j}$ corresponding $1 \sigma$ errors. $P_{J}$ is the pull of the Jarlskog invariant which, in the standard parameterization, is given by $\bar{J}=c_{12} c_{23} c_{13}^{2} s_{12} s_{23} s_{13} \sin \delta=-0.0270054$ and the corresponding $1 \sigma$ uncertainty is $\delta J=0.0106304$, for normal ordering [33]. The theoretical prediction is given by $J=\operatorname{Im}\left(U_{\mu 3} U_{\tau 3}^{*} U_{\mu 2} U_{\tau 2}^{*}\right)$, where in this expression $U$ stands for the PMNS mixing matrix. The upper bound $m_{1}+m_{2}+m_{3}<0.17 \mathrm{eV}$ [33], is also imposed. Using the data from [38] ${ }^{1}$, the fit results are shown in the following tables:

\footnotetext{
${ }^{1}$ NuFIT collaboration (http://www.nu-fit.org/?q=node/211)(with SK atmospheric data).
} 


\begin{tabular}{|c|c|c|c|c|}
\hline$m_{1}(\mathrm{eV})$ & $c_{\ell}(\mathrm{eV})$ & $\phi_{1}(\mathrm{rad})$ & $\phi_{2}(\mathrm{rad})$ & $\chi_{\min }^{2}$ \\
\hline $0.00395 \pm_{0.00068}^{0.00062}$ & 523176. & 0.0190664 & 1.56122 & 12.4204 \\
\hline
\end{tabular}

TABLE I: Best fit free parameters and minimum $\chi^{2}$ function.

\begin{tabular}{|c|c|c|c|c|c|c|c|c|c|}
\hline$P_{11}$ & $P_{12}$ & $P_{13}$ & $P_{21}$ & $P_{22}$ & $P_{23}$ & $P_{31}$ & $P_{32}$ & $P_{33}$ & $P_{J}$ \\
\hline 0.428531 & -0.385085 & 0.0430767 & -0.205321 & -1.2577 & 1.91336 & 0.290472 & 1.25036 & -2.2701 & 0.0228083 \\
\hline
\end{tabular}

TABLE II: $P_{i, j}$ is the pull of the $i, j$ component of the PMNS matrix and $P_{J}$ is the pull of the Jarlskog invariant in the $\chi^{2}$ analysis. The minimum of the $\chi^{2}$ function is 12.4204 for ten observables and four parameters $\left(m_{1}, c_{\ell}, \phi_{1}, \phi_{2}\right)$. The fit goodness is $\chi^{2} /$ d.o.f $=2.07$ which is a relatively high value due to $P_{33}$ and $P_{23}$ pulls which have a deviation around $2 \sigma$ respect to their experimental values, despite this result it is still an acceptable fit.

In our $\chi^{2}$ analysis the pseudo observables are the absolute values of the PMNS matrix components and the Jarlskog invariant, with pulls $P_{i, j}$ and $P_{J}$, respectively. We do not consider correlations between them ${ }^{2}$.

Even though that a value for the fit goodness $\chi^{2} /$ d.o.f $\sim 2.07$ is not optimal, the result is acceptable. We can see that the main source of tension is related to the $\left(V_{\mathrm{PMNS}}\right)_{23}$ and $\left(V_{\mathrm{PMNS}}\right)_{33}$ components, which deviate from their experimental values by $2 \sigma$. It is important to stress that a lightest neutrino mass equal to zero and the inverse ordering of the neutrino masses, they are not favored by this texture (the same is true for the equivalent textures via weak basis transformations). It is possible that for another non-equivalent five-zero texture a realization of the inverse ordering. This subject requires a more dedicated study.

\section{FIVE TEXTURE ZEROS. SECOND CASE.}

Let us now assume that in the context of the SMRHN with the neutrinos being only Dirac type particles, there is a symmetry which produces the Hermitian mass matrices $M_{n}^{\prime}$ and $M_{\ell}^{\prime}$ with the following textures

$$
M_{n}^{\prime}=\left(\begin{array}{ccc}
0 & C_{n} & 0 \\
C_{n}^{*} & D_{n} & B_{n} \\
0 & B_{n}^{*} & A_{n}
\end{array}\right), \quad M_{\ell}^{\prime}=\left(\begin{array}{ccc}
0 & C_{\ell} & 0 \\
C_{\ell}^{*} & 0 & B_{\ell} \\
0 & B_{\ell}^{*} & A_{\ell}
\end{array}\right)
$$

Let us analyze the consequences of this new pattern with three texture zeros in the charged sector and two in the neutral one. Without losing generality, it is possible to eliminate the phases of the $M_{\ell}^{\prime}$ matrix by means of a WBT, so that the $\mathrm{CP}$ violation phase only appears in the neutrino mass matrix. The algebra shows that it is possible to diagonalize the lepton sector $M_{\ell}=U_{\ell} D_{\ell} U_{\ell}^{\dagger}$ (which as in the previous section we define $\left.\left(M_{\ell}\right)_{i, j}=\left|\left(M_{\ell}^{\prime}\right)_{i, j}\right|\right)$, where $D_{\ell}=$ Diag. $\left(m_{e},-m_{\mu}, m_{\tau}\right)$, in order to take full advantage of using the following unitary matrix

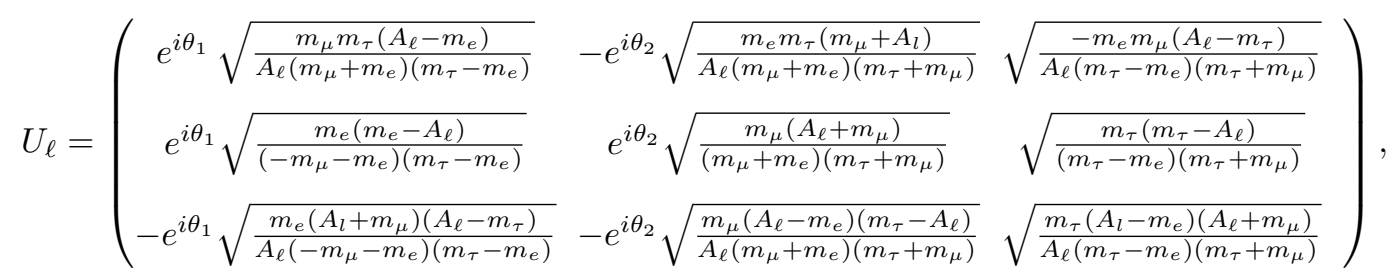

where $\theta_{1}$ and $\theta_{2}$ are arbitrary phases and $A_{\ell}=m_{e}-m_{\mu}+m_{\tau}$. Even though the phases, $\theta_{1}$ and $\theta_{2}$, in the rotation matrix of $U_{l}$ are no CP phases (they can be absorbed in the fields), these phases are quite useful to match our

\footnotetext{
2 The collaborations report correlation effects between observables, in our case, the components of the PMNS matrix are the result of a global fit. However, in phenomenology is a common practice to use pseudo observables.
} 
theoretical expression for the PMNS matrix with the standard convention [39]. To get the three texture zeros in the lepton mass matrix the following relations are also necessary

$$
\left|B_{\ell}\right|=\sqrt{\frac{\left(A_{\ell}-m_{e}\right)\left(A_{\ell}+m_{\mu}\right)\left(m_{\tau}-A_{\ell}\right)}{A_{\ell}}} \quad \text { and } \quad\left|C_{\ell}\right|=\sqrt{\frac{m_{e} m_{\mu} m_{\tau}}{A_{\ell}}} .
$$

For the neutrino sector we are subject to the condition $U_{\ell}^{\dagger} U_{n}=V_{\mathrm{PMNS}}$ and, necessarily, the diagonalizing matrix must be given by $U_{n}=U_{\ell} V_{\mathrm{PMNS}}$, so that the relation between the mass matrix in the weak basis and the diagonal matrix $D_{n}$ in the mass space is

$$
M_{n}^{\prime}=\left(\begin{array}{ccc}
0 & C_{n} & 0 \\
C_{n}^{*} & D_{n} & B_{n} \\
0 & B_{n}^{*} & A_{n}
\end{array}\right)=U_{\ell}\left(V_{\mathrm{PMNS}}\right) D_{n}\left(V_{\mathrm{PMNS}}\right)^{\dagger} U_{\ell}^{\dagger} \equiv U_{n} D_{n} U_{n}^{\dagger} .
$$

For this second case the only free parameters are: $m_{1}$ from the diagonal matrix $D_{n}=$ Diag. $\left(m_{1},-m_{2}, m_{3}\right)$, and $\theta_{1}$ and $\theta_{2}$ from $U_{\ell}$. This result is important since we can interpret the neutrino masses as predictions associated with the texture of the mass matrices. From these expressions we can obtain useful relations by identifying $U_{\ell}$ with the weak basis transformation $U$ in equation (2) [35].

\section{A. Numerical results}

For this second case, when solving numerically to obtain the texture for the neutrino mass matrix in the normal hierarchy for the neutrino masses, we obtain

$$
\begin{aligned}
& m_{1}=(0.00354 \pm 0.00088) \mathrm{eV}, \\
& m_{2}=(0.00930 \pm 0.00036) \mathrm{eV}, \\
& m_{3}=(0.05040 \pm 0.00030) \mathrm{eV} .
\end{aligned}
$$

In our numerical analysis the main source of uncertainty comes from the $\mathrm{CP}$ violation phase, result which makes sense since in the lepton sector this parameter has not been determined with good precision. The associated numerical entries for lepton mass matrices with five texture zeros are (in MeV): $A_{n}=0.0251821, B_{n}=(-0.0122955+0.0244187 i)$, $C_{n}=(0.00427236+0.00689527 i), D_{n}=0.0194623, A_{\ell}=1671.71,\left|B_{\ell}\right|=432.237,\left|C_{\ell}\right|=7.57544$, and phases $\theta_{1}=0.154895$ and $\theta_{2}=2.01797$. The phases of $B_{\ell}$ and $C_{\ell}$ were absorbed in $B_{n}$ and $C_{n}$ by means of a redefinition, through a WBT, in a previous step.

By construction, the WBT formalism reproduces the mixing matrix, the mass of charged leptons and the neutrino mass squared differences. We used, as input parameters, the central values of the global fit reported by the Nu-FIT collaboration (with SK atmospheric data) [38]. When comparing with the method of least squares in the WBT there are no deviations from the experimental values, see Table III A.

\begin{tabular}{|c|c|c|c|c|c|c|c|c|}
\hline$\theta_{12}\left({ }^{\circ}\right)$ & $\theta_{23}\left({ }^{\circ}\right)$ & $\theta_{13}\left({ }^{\circ}\right)$ & $\delta_{C P}\left(^{\circ}\right)$ & $\delta m_{21}^{2}\left(\mathrm{eV}^{2}\right)$ & $\delta m_{31}^{2}\left(\mathrm{eV}^{2}\right)$ & $m_{e}(\mathrm{MeV})$ & $m_{\mu}(\mathrm{MeV})$ & $m_{\tau}(\mathrm{MeV})$ \\
\hline & & & & & & & & \\
33.82 & 48.6 & 8.60 & 221 & $7.39 \times 10^{-5}$ & $2.528 \times 10^{-3}$ & 0.510999 & 105.658 & 1776.86 \\
\hline
\end{tabular}

TABLE III: Output values in our analysis.

\section{CONCLUSIONS}

In this analysis, we explore the consequences of extending the SM with three right-handed neutrinos that allow nine additional complex Dirac mass terms for the neutral lepton sector, and excluding the possibility of having bare Majorana masses. Two different five texture zeros for the Hermitian lepton mass matrices were considered, one with three zeros in the neutral sector and two in the charged sector, and the other one with two texture zeros in the neutral 
sector and three in the charged one. In order to have reliable results we have used two different approaches assuming for both a normal ordering for the neutrino physical masses.

Counting the degrees of freedom in the lepton sector, and after making use of the polar theorem of matrix algebra and the consequences of the WBT, we have concluded that with five texture zeros in the Hermitian mass matrices, only one prediction can be reached. We have chosen the lightest neutrino mass for such a predicted value.

The first analysis, based on a least squares approach used to adjust the lepton masses and the mixing parameters to their corresponding experimental values, was implemented for the texture with three zeros in the neutral sector and two in the charged one. In this approach, the fit of the mixing parameters to the reported values in the literature is below two sigmas with an acceptable fit goodness. The best fit for the lightest neutrino mass in this case was $m_{1} \approx\left(3.9 \pm_{0.8}^{0.6}\right) \times 10^{-3} \mathrm{eV}$, which is similar to reported values starting from other assumptions [21-23].

The second analysis was a purely algebraic and numerical study, based on the WBT approach. It was implemented for the texture with three zeros in the charged lepton sector and two in the neutral one. The prediction now for the lightest neutrino mass was $(3.5 \pm 0.9) \times 10^{-3} \mathrm{eV}$., in agreement with the previous result.

The two different five texture-zeros proposed in expressions (4) and (12) are not equivalent in the sense that there is not a WBT relating them.

\section{Acknowledgments}

This work was partially supported by VIPRI in the Universidad de Nariño, Approval Contract No. 024 and 160 , Project Code: 1048 and 1928. R.H.B and L.M. thanks the "Centro de Laboratorios de investigación parque i-ITM".

[1] J. F. Donoghue, E. Golowich and B. R. Holstein, Camb. Monogr. Part. Phys. Nucl. Phys. Cosmol. 2, 1 (1992) [Camb. Monogr. Part. Phys. Nucl. Phys. Cosmol. 35 (2014)]. doi:10.1017/CBO9780511524370

[2] E. Peinado, M. Reig, R. Srivastava and J. W. F. Valle, arXiv:1910.02961 [hep-ph].

[3] P. Minkowski, Phys. Lett. 67B, 421 (1977). doi:10.1016/0370-2693(77)90435-X

[4] M. Gell-Mann, P. Ramond and R. Slansky, Conf. Proc. C 790927, 315 (1979) [arXiv:1306.4669 [hep-th]].

[5] T. Yanagida, Prog. Theor. Phys. 64, 1103 (1980). doi:10.1143/PTP.64.1103

[6] S. L. Glashow, NATO Sci. Ser. B 61, 687 (1980). doi:10.1007/978-1-4684-7197-7_15

[7] R. N. Mohapatra and G. Senjanovic, Phys. Rev. Lett. 44, 912 (1980). doi:10.1103/PhysRevLett.44.912

[8] J. Schechter and J. W. F. Valle, Phys. Rev. D 22, 2227 (1980). doi:10.1103/PhysRevD.22.2227

[9] G. Ahuja, M. Gupta, M. Randhawa and R. Verma, Phys. Rev. D 79, 093006 (2009) doi:10.1103/PhysRevD.79.093006 [arXiv:0904.4534 [hep-ph]].

[10] X. w. Liu and S. Zhou, Int. J. Mod. Phys. A 28, 1350040 (2013) doi:10.1142/S0217751X13500401 [arXiv:1211.0472 [hep$\mathrm{ph}]$.

[11] R. Verma, Phys. Rev. D 88, 111301 (2013) doi:10.1103/PhysRevD.88.111301 [arXiv:1311.7524 [hep-ph]].

[12] P. O. Ludl and W. Grimus, JHEP 1407, 090 (2014) Erratum: [JHEP 1410, 126 (2014)] doi:10.1007/JHEP07(2014)090, 10.1007/JHEP10(2014)126 [arXiv:1406.3546 [hep-ph]].

[13] R. Verma, Int. J. Mod. Phys. A 29, no. 21, 1444009 (2014) doi:10.1142/S0217751X14440096 [arXiv:1406.0640 [hep-ph]].

[14] P. Fakay, S. Sharma, G. Ahuja and M. Gupta, PTEP 2014, no. 2, $023 B 03$ (2014) doi:10.1093/ptep/ptu005 [arXiv:1401.8121 [hep-ph]].

[15] L. M. Cebola, D. Emmanuel-Costa and R. G. Felipe, Phys. Rev. D 92, no. 2, 025005 (2015) doi:10.1103/PhysRevD.92.025005 [arXiv:1504.06594 [hep-ph]].

[16] R. R. Gautam, M. Singh and M. Gupta, Phys. Rev. D 92, no. 1, 013006 (2015) doi:10.1103/PhysRevD.92.013006 [arXiv:1506.04868 [hep-ph]].

[17] G. Ahuja, S. Sharma, P. Fakay and M. Gupta, Mod. Phys. Lett. A 30, no. $34, \quad 1530025$ (2015) doi:10.1142/S0217732315300256 [arXiv:1604.03339 [hep-ph]].

[18] M. Singh, Nucl. Phys. B 931, 446 (2018) doi:10.1016/j.nuclphysb.2018.05.004 [arXiv:1804.00842 [hep-ph]].

[19] G. Ahuja and M. Gupta, Int. J. Mod. Phys. A 33, no. 31, 1844032 (2018). doi:10.1142/S0217751X18440323

[20] S. Dell'Oro, S. Marcocci, M. Viel and F. Vissani, Adv. High Energy Phys. 2016, 2162659 (2016) doi:10.1155/2016/2162659 [arXiv:1601.07512 [hep-ph]].

[21] H. Fritzsch, Mod. Phys. Lett. A 30, no. 16, 1530012 (2015) doi:10.1142/S0217732315300128 [arXiv:1503.01857 [hep-ph]].

[22] H. Fritzsch, Subnucl. Ser. 53, 201 (2017) doi:10.1142/9789813208292_0006 [arXiv:1503.07927 [hep-ph]].

[23] H. Fritzsch, Mod. Phys. Lett. A 31, no. 15, 1630014 (2016). doi:10.1142/S0217732316300147

[24] S. Antusch, O. J. Eyton-Williams and S. F. King, JHEP 0508, 103 (2005) doi:10.1088/1126-6708/2005/08/103 [hep$\mathrm{ph} / 0505140]$.

[25] P. Langacker, Ann. Rev. Nucl. Part. Sci. 62, 215 (2012) doi:10.1146/annurev-nucl-102711-094925 [arXiv:1112.5992 [hep$\mathrm{ph}]$. 
[26] G. Dvali and L. Funcke, Phys. Rev. D 93, no. 11, 113002 (2016) doi:10.1103/PhysRevD.93.113002 [arXiv:1602.03191 [hep-ph]].

[27] G. Barenboim, J. Turner and Y. L. Zhou, arXiv:1909.04675 [hep-ph].

[28] C. Broggini, C. Giunti and A. Studenikin, Adv. High Energy Phys. 2012, 459526 (2012) doi:10.1155/2012/459526 [arXiv:1207.3980 [hep-ph]].

[29] M. Kobayashi and T. Maskawa, Prog. Theor. Phys. 49, 652 (1973). doi:10.1143/PTP.49.652

[30] L. Maiani, Phys. Lett. 62B, 183 (1976). doi:10.1016/0370-2693(76)90500-1

[31] G. C. Branco, L. Lavoura and F. Mota, Phys. Rev. D 39, 3443 (1989). doi:10.1103/PhysRevD.39.3443

[32] G. C. Branco, D. Emmanuel-Costa and R. Gonzalez Felipe, Phys. Lett. B 477, 147 (2000) doi:10.1016/S03702693(00)00193-3 [hep-ph/9911418].

[33] M. Tanabashi et al. [Particle Data Group], Phys. Rev. D 98, no. 3, 030001 (2018). doi:10.1103/PhysRevD.98.030001

[34] H. Fusaoka and Y. Koide, Phys. Rev. D 57, 3986 (1998) doi:10.1103/PhysRevD.57.3986 [hep-ph/9712201].

[35] Y. Giraldo, Phys. Rev. D 86, 093021 (2012) doi:10.1103/PhysRevD.86.093021 [arXiv:1110.5986 [hep-ph]].

[36] Y. Giraldo, arXiv:1511.08858 [hep-ph].

[37] R. Verma, Adv. High Energy Phys. 2016, 2094323 (2016) doi:10.1155/2016/2094323 [arXiv:1607.00958 [hep-ph]].

[38] I. Esteban, M. C. Gonzalez-Garcia, A. Hernandez-Cabezudo, M. Maltoni and T. Schwetz, JHEP 1901, 106 (2019) doi:10.1007/JHEP01(2019)106 [arXiv:1811.05487 [hep-ph]].

[39] A. Rasin, hep-ph/9708216. 